\title{
Flow of a Second-Grade Fluid between Eccentric Rotating Porous Disks in the Presence of a Magnetic Field
}

\author{
H. Volkan Ersoy \\ Department of Mechanical Engineering, Yildiz Technical University, Istanbul, Turkey \\ Email: ersoyhv@yahoo.com
}

How to cite this paper: Ersoy, H.V. (2018) Flow of a Second-Grade Fluid between Eccentric Rotating Porous Disks in the Presence of a Magnetic Field. Open Journal of Applied Sciences, 8, 159-169. https://doi.org/10.4236/ojapps.2018.85013

Received: April 1, 2018

Accepted: May 27, 2018

Published: May 30, 2018

Copyright $\odot 2018$ by author and Scientific Research Publishing Inc. This work is licensed under the Creative Commons Attribution International License (CC BY 4.0).

http://creativecommons.org/licenses/by/4.0/

\section{(c) (i) Open Access}

\begin{abstract}
This paper is concerned with the steady flow of a second-grade fluid between two porous disks rotating eccentrically under the effect of a magnetic field. A perturbation solution for the velocity field is presented under the assumption that the second-grade fluid parameter $\beta$ is small. It is also studied the effect of all the parameters on the horizontal force per unit area exerted by the fluid on the disks. It is found that the $x$ - and $y$-components of the force increase and decrease, respectively, when the second-grade fluid parameter $\beta$ and the Hartmann number $M$ increase. It is seen that the forces in the $x$ - and $y$-directions on the top disk increase with the increase of the suction/injection velocity parameter $P$ but those on the bottom disk decrease. It is shown that the force acting on the top disk is greater than that acting on the bottom disk in view of the axial velocity in the positive $z$-direction. It is observed that the increase in the Reynolds number $R$ leads to a rise in the horizontal force.
\end{abstract}

\section{Keywords}

Second-Grade Fluid, Eccentric Rotating Porous Disks, Magnetohydrodynamics, Perturbation Solution

\section{Introduction}

The orthogonal rheometer consisting of two parallel disks rotating with the same angular velocity about distinct axes was developed by Maxwell and Chartoff [1]. An exact solution corresponding to the flow of a Newtonian fluid in this instrument was obtained by Abbott and Walters [2]. Rajagopal and Gupta [3] were the first to obtain an exact solution for a second-grade fluid and also studied the stability of the flow. Rajagopal [4] also studied the problem for a second order fluid 
whose normal stress moduli do not obey the relations $\alpha_{1} \geq 0$ and $\alpha_{1}+\alpha_{2}=0$. Rajagopal [5] showed that this motion is one with constant principal relative stretch history.

The steady flow between eccentric rotating disks under the effect of a magnetic field has been studied by many researchers. Mohanty [6] was the first to obtain an exact solution to the MHD flow for a Newtonian fluid. Rao and Rao [7] investigated the MHD flow for a second-grade fluid. Ersoy [8] studied the MHD flow in the case of an Oldroyd-B fluid. Guria et al. [9] examined the effect of the heat transfer for a Newtonian fluid in the presence of a magnetic field for porous disks. Siddiqui et al. [10] investigated the MHD flow of a Burger's fluid. Ersoy [11] studied the flow of a Maxwell fluid under the effect of a magnetic field when the disks are porous.

The aim of this paper is to study the steady flow of an incompressible second-grade fluid between two insulated and porous disks rotating with the same angular velocity about two parallel axes under the effect of a magnetic field. It is a well-known fact that the governing equations of non-Newtonian fluids are in general of higher order than the Navier-Stokes equations. Even so, it is possible to find an exact solution for a second-grade fluid in this geometry when the disks are non-porous [7]. However, the order of the governing equation for porous disks exceeds the number of boundary conditions. The perturbation solution is obtained by taking the second-grade fluid parameter $\beta$ as the perturbation parameter. The velocity field and the horizontal components of the dimensionless force per unit area exerted by the fluid on the top and bottom disks are constructed for small values of the second-grade fluid parameter.

\section{Description of the Problem}

Let us consider an incompressible second-grade fluid between two porous and insulated disks located at $z= \pm h$. The top and bottom disks rotate about the $z^{\prime}$ and $z^{\prime \prime}$-axes with the same angular velocity $\Omega$, respectively. The distance between the axes of rotation is defined by $2 \ell$ in the $y$-direction. A uniform magnetic induction $\boldsymbol{B}_{0}$ is applied in the positive $z$-direction. It is assumed that the magnetic Reynolds number for the flow is very small so that the induced magnetic field is negligible in comparison with the applied magnetic field. A uniform suction and injection are applied perpendicular to the top and bottom disks, respectively. The schematic configuration of the problem is illustrated in Figure 1. Therefore, the appropriate boundary conditions for the velocity field are

$$
\begin{gathered}
u=-\Omega(y-\ell), v=\Omega x, w=w_{0} \text { at } z=h, \\
u=-\Omega(y+\ell), v=\Omega x, w=w_{0} \text { at } z=-h, \\
u=-\Omega y, v=\Omega x, w=w_{0} \text { at } z=0,
\end{gathered}
$$

where $u, V, W$ denote the velocity components along the $x, y, z$-directions, respectively. We look for a solution of the velocity field of the form

$$
u=-\Omega y+f(z), v=\Omega x+g(z), \quad w=w_{0} .
$$




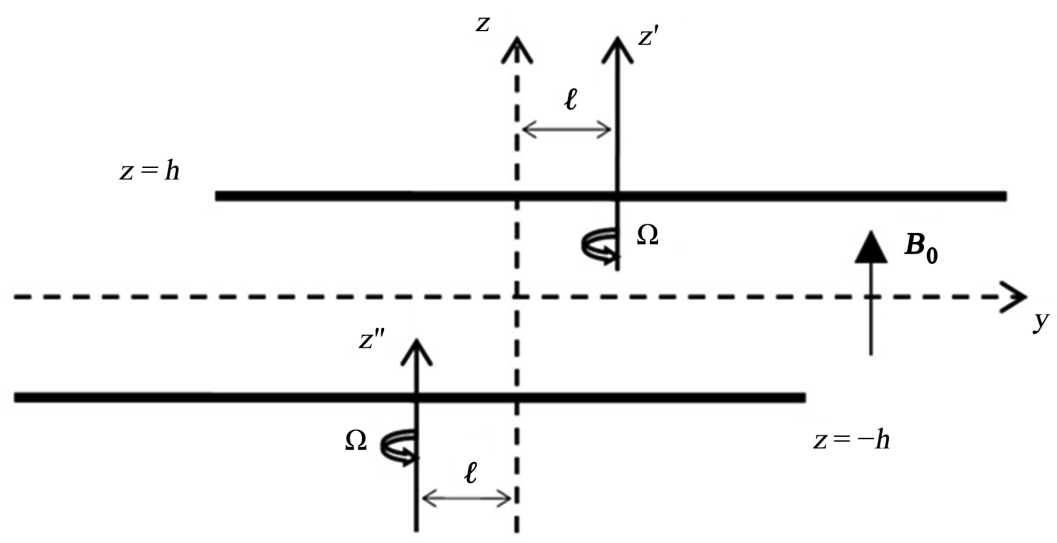

Figure 1. Flow geometry.

\section{Solution of the Problem}

The equations governing the flow are

$$
\begin{gathered}
\rho \dot{\boldsymbol{v}}=\nabla \cdot \boldsymbol{T}+\boldsymbol{J} \times \boldsymbol{B}, \\
\nabla \cdot \boldsymbol{v}=0,
\end{gathered}
$$

where $\rho$ is the density, $\boldsymbol{v}$ the velocity vector, $\boldsymbol{J}=\left(J_{x}, J_{y}, J_{z}\right)$ the current density, $\boldsymbol{B}$ the magnetic induction and the overdot denotes material time differentiation. The velocity field in Equation (4) is compatible with the continuity Equation (6).

The stress $\boldsymbol{T}$ in a homogeneous second-grade fluid is given by [12]

$$
\boldsymbol{T}=-p \boldsymbol{I}+\mu \boldsymbol{A}_{1}+\alpha_{1} \boldsymbol{A}_{2}+\alpha_{2} \boldsymbol{A}_{1}^{2},
$$

where $p$ is the pressure, $\boldsymbol{I}$ the identity tensor, $\mu$ the dynamic viscosity coefficient, $\alpha_{1}$ and $\alpha_{2}$ the normal stress moduli. $A_{1}$ and $A_{2}$ stand for the first two Rivlin-Ericksen tensors defined through

$$
\boldsymbol{A}_{1}=\operatorname{grad} \boldsymbol{v}+(\operatorname{grad} v)^{\mathrm{T}}
$$

and

$$
\boldsymbol{A}_{2}=\dot{\boldsymbol{A}}_{1}+\boldsymbol{A}_{1}(\operatorname{grad} \boldsymbol{v})+(\operatorname{grad} \boldsymbol{v})^{\mathrm{T}} \boldsymbol{A}_{1}
$$

The coefficients $\mu, \alpha_{1}$, and $\alpha_{2}$ must satisfy

$$
\mu \geq 0, \alpha_{1} \geq 0, \alpha_{1}+\alpha_{2}=0 .
$$

We refer the reader to [13] [14] [15] for more information about second-grade fluids.

Inserting Equation (4) into Equations (5), (7)-(9), we obtain

$$
\begin{gathered}
\frac{\partial p}{\partial x}=\alpha_{1}\left(w_{0} f^{\prime \prime \prime}+\Omega g^{\prime \prime}\right)+\mu f^{\prime \prime}-\rho w_{0} f^{\prime}+\rho \Omega(\Omega x+g)+B_{0} J_{y}, \\
\frac{\partial p}{\partial y}=\alpha_{1}\left(w_{0} g^{\prime \prime \prime}-\Omega f^{\prime \prime}\right)+\mu g^{\prime \prime}-\rho w_{0} g^{\prime}+\rho \Omega(\Omega y-f)-B_{0} J_{x}, \\
\frac{\partial p}{\partial z}=\left(2 \alpha_{1}+\alpha_{2}\right)\left(2 f^{\prime} f^{\prime \prime}+2 g^{\prime} g^{\prime \prime}\right),
\end{gathered}
$$


where a prime denotes differentiation with respect to $z$. Using $\boldsymbol{J}=\sigma(\boldsymbol{E}+\boldsymbol{v} \times \boldsymbol{B})$, we have

$$
J_{x}=\sigma\left(E_{x}+v B_{0}\right), J_{y}=\sigma\left(E_{y}-u B_{0}\right), J_{z}=\sigma E_{z},
$$

where $\sigma$ is the electrical conductivity of the fluid, $\boldsymbol{E}=\left(E_{x}, E_{y}, E_{z}\right)$ the electric field and $B_{0}$ the magnitude of the applied magnetic induction $\boldsymbol{B}_{0}$. Since the disks are insulated, we obtain $J_{z}=0$ and $E_{z}=0$. Using $\nabla \times \boldsymbol{E}=\mathbf{0}$, we have

$$
\begin{gathered}
\alpha_{1}\left(w_{0} f^{\prime \prime \prime}+\Omega g^{\prime \prime}\right)+\mu f^{\prime \prime}-\rho w_{0} f^{\prime}+\rho \Omega g-\sigma B_{0}^{2} f=C_{1}, \\
\alpha_{1}\left(w_{0} g^{\prime \prime \prime}-\Omega f^{\prime \prime}\right)+\mu g^{\prime \prime}-\rho w_{0} g^{\prime}-\rho \Omega f-\sigma B_{0}^{2} g=C_{2},
\end{gathered}
$$

where $C_{1}$ and $C_{2}$ are constants. Combining Equations (15)-(16) and using $F(z)=f(z)+i g(z)$, we get

$$
\alpha_{1} w_{0} F^{\prime \prime \prime}+\left(\mu-i \alpha_{1} \Omega\right) F^{\prime \prime}-\rho w_{0} F^{\prime}-\left(\sigma B_{0}^{2}+i \rho \Omega\right) F=C,
$$

where $C$ is a constant. The boundary conditions for $F(z)$ are as follows:

$$
F(h)=\Omega \ell, \quad F(-h)=-\Omega \ell, \quad F(0)=0 .
$$

Introducing the dimensionless quantities,

$$
\begin{gathered}
\bar{F}(\zeta)=\frac{F(z)}{\Omega \ell}, \quad \zeta=\frac{z}{h}, M=\sqrt{\frac{\sigma}{\mu}} B_{0} h, \quad \beta=\frac{\alpha_{1} \Omega}{\mu}, \\
P=\frac{\rho w_{0} h}{\mu}, \quad R=\frac{\rho \Omega h^{2}}{\mu},
\end{gathered}
$$

the governing Equation (17) and the conditions (18) reduce to the following dimensionless forms:

$$
\begin{gathered}
\beta P \bar{F}^{\prime \prime \prime}+(1-i \beta) R \bar{F}^{\prime \prime}-P R \bar{F}^{\prime}-\left(M^{2}+i R\right) R \bar{F}=\bar{K}, \\
\bar{F}(1)=1, \quad \bar{F}(0)=0, \quad \bar{F}(-1)=-1,
\end{gathered}
$$

where a prime denotes differentiation with respect to $\zeta$, and $\bar{K}$ is a constant.

We look for a perturbation solution for small values of the second-grade fluid parameter $\beta$ as follows:

$$
\begin{gathered}
\bar{F}(\zeta)=\bar{F}_{0}(\zeta)+\beta \bar{F}_{1}(\zeta)+\beta^{2} \bar{F}_{2}(\zeta)+O\left(\beta^{3}\right), \\
\bar{K}=\bar{K}_{0}+\beta \bar{K}_{1}+\beta^{2} \bar{K}_{2}+O\left(\beta^{3}\right),
\end{gathered}
$$

where $\bar{K}_{0}, \bar{K}_{1}$ and $\bar{K}_{2}$ are constants. Substituting Equations (22)-(23) into Equations (20)-(21) and equating the coefficients of different powers of $\beta$, we obtain

$$
\begin{gathered}
R \bar{F}_{0}^{\prime \prime}-P R \bar{F}_{0}^{\prime}-\left(M^{2}+i R\right) R \bar{F}_{0}=\bar{K}_{0}, \\
P \bar{F}_{0}^{\prime \prime \prime}+R \bar{F}_{1}^{\prime \prime}-i R \bar{F}_{0}^{\prime \prime}-P R \bar{F}_{1}^{\prime}-\left(M^{2}+i R\right) R \bar{F}_{1}=\bar{K}_{1}, \\
P \bar{F}_{1}^{\prime \prime \prime}+R \bar{F}_{2}^{\prime \prime}-i R \bar{F}_{1}^{\prime \prime}-P R \bar{F}_{2}^{\prime}-\left(M^{2}+i R\right) R \bar{F}_{2}=\bar{K}_{2},
\end{gathered}
$$

and

$$
\bar{F}_{0}(1)=1, \quad \bar{F}_{0}(0)=0, \quad \bar{F}_{0}(-1)=-1,
$$




$$
\begin{array}{ccc}
\bar{F}_{1}(1)=0, & \bar{F}_{1}(0)=0, & \bar{F}_{1}(-1)=0, \\
\bar{F}_{2}(1)=0, & \bar{F}_{2}(0)=0, & \bar{F}_{2}(-1)=0 .
\end{array}
$$

The solution of Equation (24) with Equation (27) is

$$
\bar{F}_{0}(\zeta)=\bar{A}_{0} \exp (a \zeta)+\bar{B}_{0} \exp (b \zeta)+\bar{C}_{0},
$$

where

$$
\begin{gathered}
a=\frac{P+\sqrt{P^{2}+4\left(M^{2}+i R\right)}}{2}, \quad b=\frac{P-\sqrt{P^{2}+4\left(M^{2}+i R\right)}}{2}, \\
\bar{A}_{0}=\frac{1-\cosh b}{\sinh a-\sinh b-\sinh (a-b)}, \quad \bar{B}_{0}=\frac{\cosh a-1}{\sinh a-\sinh b-\sinh (a-b)}, \\
\bar{C}_{0}=\frac{\cosh b-\cosh a}{\sinh a-\sinh b-\sinh (a-b)} .
\end{gathered}
$$

The solution of Equation (25) with Equation (28) is

$$
\bar{F}_{1}=\left(\bar{A}_{1}+\bar{B}_{1} \zeta\right) \exp (a \zeta)+\left(\bar{C}_{1}+\bar{D}_{1} \zeta\right) \exp (b \zeta)+\bar{E}_{1},
$$

where

$$
\begin{gathered}
\bar{A}_{1}=\bar{A}_{0} \bar{D}_{1}+\bar{E}_{0} \bar{B}_{1}, \quad \bar{E}_{0}=\frac{\cosh (a-b)-\cosh a}{\sinh a-\sinh b-\sinh (a-b)}, \\
\bar{B}_{1}=\frac{a^{2} \bar{A}_{0}}{P-2 a}\left(\frac{P a}{R}-i\right), \bar{C}_{1}=\bar{B}_{0} \bar{B}_{1}+\bar{H}_{0} \bar{D}_{1}, \\
\bar{H}_{0}=\frac{\cosh b-\cosh (a-b)}{\sinh a-\sinh b-\sinh (a-b)}, \bar{D}_{1}=\frac{b^{2} \bar{B}_{0}}{P-2 b}\left(\frac{P b}{R}-i\right), \\
\bar{E}_{1}=\bar{I}_{0}\left(\bar{B}_{1}-\bar{D}_{1}\right), \bar{I}_{0}=\frac{1-\cosh (a-b)}{\sinh a-\sinh b-\sinh (a-b)} .
\end{gathered}
$$

Finally, the solution of Equation (26) subject to Equation (29) is

$$
\bar{F}_{2}=\left(\bar{A}_{2}+\bar{B}_{2} \zeta+\bar{C}_{2} \zeta^{2}\right) \exp (a \zeta)+\left(\bar{D}_{2}+\bar{E}_{2} \zeta+\bar{H}_{2} \zeta^{2}\right) \exp (b \zeta)+\bar{I}_{2},
$$

where

$$
\begin{gathered}
\bar{A}_{2}=\bar{A}_{0} \bar{E}_{2}+\bar{E}_{0} \bar{B}_{2}+\bar{N}_{0} \bar{C}_{2}-\bar{P}_{0} \bar{H}_{2}, \quad \bar{N}_{0}=\frac{\sinh (a-b)-\sinh a}{\sinh a-\sinh b-\sinh (a-b)}, \\
\bar{P}_{0}=\frac{\sinh b}{\sinh a-\sinh b-\sinh (a-b)}, \quad \bar{B}_{2}=\frac{\bar{L}_{1}}{P-2 a}+\frac{\bar{L}_{2}}{(P-2 a)^{2}}, \\
\bar{L}_{1}=\frac{P a^{2}}{R}\left(a \bar{A}_{1}+3 \bar{B}_{1}\right)-i a\left(a \bar{A}_{1}+2 \bar{B}_{1}\right), \bar{L}_{2}=a^{2} \bar{B}_{1}\left(\frac{P a}{R}-i\right), \\
\bar{C}_{2}=\frac{\bar{L}_{2}}{2(P-2 a)}, \bar{D}_{2}=\bar{B}_{0} \bar{B}_{2}+\bar{H}_{0} \bar{E}_{2}+\bar{R}_{0} \bar{H}_{2}+\bar{V}_{0} \bar{C}_{2}, \\
\bar{R}_{0}=\frac{\sinh b+\sinh (a-b)}{\sinh a-\sinh b-\sinh (a-b)}, \quad \bar{V}_{0}=\frac{\sinh a}{\sinh a-\sinh b-\sinh (a-b)},
\end{gathered}
$$




$$
\begin{gathered}
\bar{E}_{2}=\frac{\bar{L}_{3}}{P-2 b}+\frac{\bar{L}_{4}}{(P-2 b)^{2}}, \bar{L}_{3}=\frac{P b^{2}}{R}\left(b \bar{C}_{1}+3 \bar{D}_{1}\right)-i b\left(b \bar{C}_{1}+2 \bar{D}_{1}\right), \\
\bar{L}_{4}=b^{2} \bar{D}_{1}\left(\frac{P b}{R}-i\right), \bar{H}_{2}=\frac{\bar{L}_{4}}{2(P-2 b)}, \\
\bar{I}_{2}=\bar{I}_{0}\left(\bar{B}_{2}-\bar{E}_{2}\right)-\bar{U}_{0}\left(\bar{C}_{2}+\bar{H}_{2}\right), \bar{U}_{0}=\frac{\sinh (a-b)}{\sinh a-\sinh b-\sinh (a-b)} .
\end{gathered}
$$

The shear stress components $T_{x z}$ and $T_{y z}$ in the fluid are presented in the dimensionless complex form as follows:

$$
\bar{T}_{x z}+i \bar{T}_{y z}=(1-i \beta) \bar{F}^{\prime}+\frac{\beta P}{R} \bar{F}^{\prime \prime},
$$

where

$$
\bar{T}_{x z}=\frac{T_{x z}}{\mu \Omega \ell / h}, \quad \bar{T}_{y z}=\frac{T_{y z}}{\mu \Omega \ell / h} .
$$

For the small values of the second-grade fluid parameter, we obtain

$$
\bar{T}_{x z}+i \bar{T}_{y z}=\bar{G}_{0}(\zeta)+\beta \bar{G}_{1}(\zeta)+\beta^{2} \bar{G}_{2}(\zeta)+O\left(\beta^{3}\right),
$$

where

$$
\begin{gathered}
\bar{G}_{0}(\zeta)=a \bar{A}_{0} \exp (a \zeta)+b \bar{B}_{0} \exp (b \zeta), \\
\bar{G}_{1}(\zeta)=\left[\bar{G}_{11}+\bar{G}_{12} \zeta\right] \exp (a \zeta)+\left[\bar{G}_{13}+\bar{G}_{14} \zeta\right] \exp (b \zeta), \\
\bar{G}_{11}=\left(\frac{P a^{2}}{R}-i a\right) \bar{A}_{0}+a \bar{A}_{1}+\bar{B}_{1}, \\
\bar{G}_{12}=a \bar{B}_{1}, \\
\bar{G}_{13}=\left(\frac{P b^{2}}{R}-i b\right) \bar{B}_{0}+b \bar{C}_{1}+\bar{D}_{1}, \\
\bar{G}_{2}(\zeta)=\left[\bar{G}_{21}+\bar{G}_{22} \zeta+\bar{G}_{23} \zeta^{2}\right] \exp (a \zeta)+\left[\bar{G}_{24}+\bar{G}_{25} \zeta+\bar{G}_{26} \zeta^{2}\right] \exp (b \zeta), \\
\bar{G}_{21}=\left(\frac{P a^{2}}{R}-i a\right) \bar{A}_{1}+\left(\frac{2 P a}{R}-i\right) \bar{B}_{1}+a \bar{A}_{2}+\bar{B}_{2}, \\
\bar{G}_{22}=\left(\frac{P a^{2}}{R}-i a\right) \bar{B}_{1}+a \bar{B}_{2}+2 \bar{C}_{2}, \\
\bar{G}_{23}=a \bar{C}_{2}, \\
\bar{G}_{24}=\left(\frac{P b^{2}}{R}-i b\right) \bar{C}_{1}+\left(\frac{2 P b}{R}-i\right) \bar{D}_{1}+b \bar{D}_{2}+\bar{E}_{2}, \\
\bar{G}_{25}=\left(\frac{P b^{2}}{R}-i b\right) \bar{D}_{1}+b \bar{E}_{2}+2 \bar{H}_{2}, \\
\bar{G}_{26}=b \bar{H}_{2} .
\end{gathered}
$$




\section{Results and Discussions}

The present flow is defined by the superposition of a helical motion and a rigid body translation that is different from plane to plane in each $z=$ constant plane. The axial component of velocity chosen in the positive $z$-direction is constant as a result of the continuity equation. Thus, the top and bottom disks are subjected to uniform suction and injection, respectively. The velocity field is presented by obtaining the functions $\bar{f}(\zeta)$ and $\bar{g}(\zeta)$ that represent the dimensionless $x$ and $y$-components of the translational velocity. The variations of $\bar{f}(\zeta)$ and $\bar{g}(\zeta)$ with the Hartmann number $M$, the second-grade fluid parameter $\beta$, the suction/injection velocity parameter $P$, the Reynolds number $R$ are drawn in Figures 2-5.

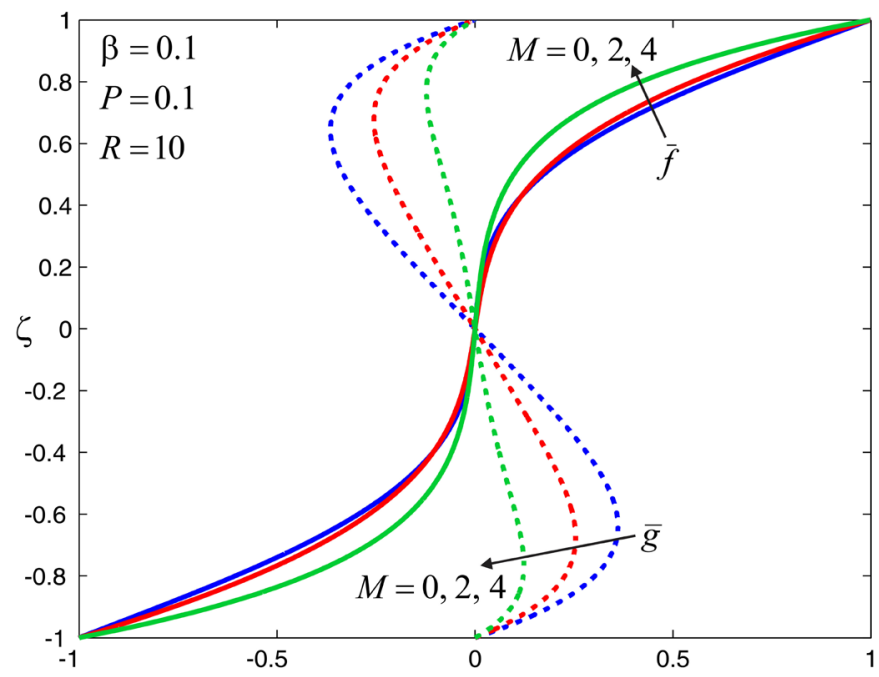

Figure 2. Variations of $\bar{f}(\zeta)$ and $\bar{g}(\zeta)$ for $M=0,2,4$ $(\beta=0.1, P=0.1, R=10)$.

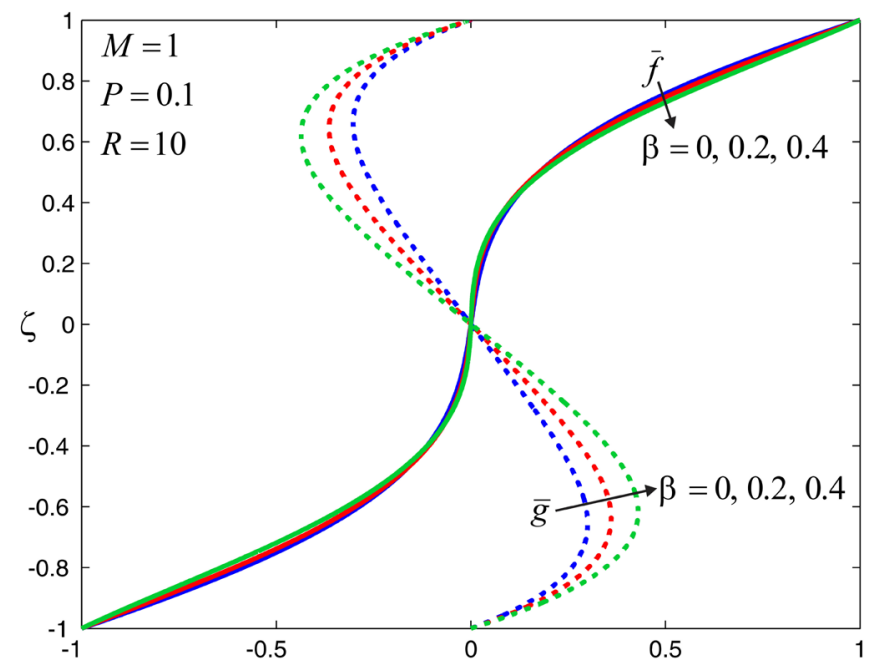

Figure 3. Variations of $\bar{f}(\zeta)$ and $\bar{g}(\zeta)$ for $\beta=0,0.2,0.4$ ( $M=1, P=0.1, R=10)$. 


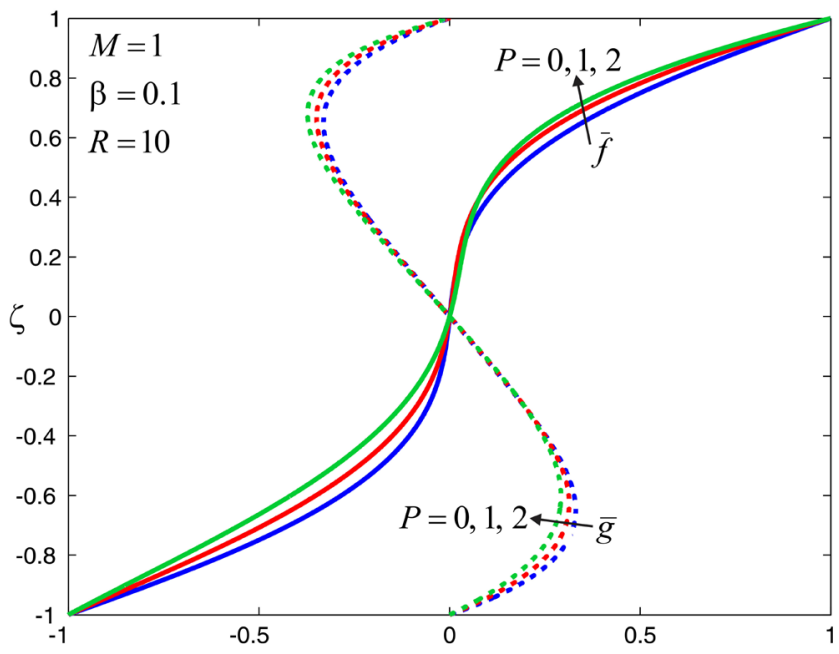

Figure 4. Variations of $\bar{f}(\zeta)$ and $\bar{g}(\zeta)$ for $P=0,1,2$ $(M=1, \beta=0.1, R=10)$.

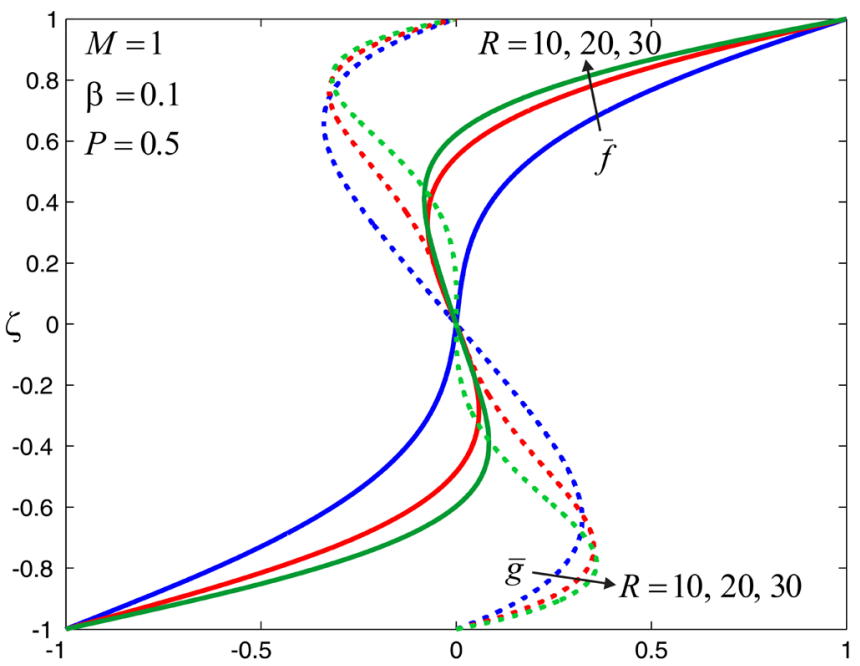

Figure 5. Variations of $\bar{f}(\zeta)$ and $\bar{g}(\zeta)$ for $R=10,20,30$ $(M=1, \beta=0.1, P=0.5)$.

The determination of the horizontal force acting on the disks is also important. $\left(\bar{T}_{x z}\right)_{\zeta= \pm 1}$ and $\left(\bar{T}_{y z}\right)_{\zeta= \pm 1}$ represent the $x$ - and $y$-components of the dimensionless force per unit area exerted by the top and bottom disks on the fluid, respectively. When the disks are non-porous, it is clear that the $x$ - and $y$-components of the force on the top disk are equal to those on the bottom disk (i.e., $\left(\bar{T}_{x z}\right)_{\zeta=1}=\left(\bar{T}_{x z}\right)_{\zeta=-1}$ and $\left.\left(\bar{T}_{y z}\right)_{\zeta=1}=\left(\bar{T}_{y z}\right)_{\zeta=-1}\right)$. In view of the fact that the axial velocity of the fluid is chosen in the pozitive $z$-direction, the components of the horizontal force on the top disk are larger than those on the bottom disk (i.e., $\left(\bar{T}_{x z}\right)_{\zeta=1}>\left(\bar{T}_{x z}\right)_{\zeta=-1}$ and $\left.\left(\bar{T}_{y z}\right)_{\zeta=1}>\left(\bar{T}_{y z}\right)_{\zeta=-1}\right)$. The effects of all the parameters on $\left(\bar{T}_{x z}\right)_{\zeta= \pm 1}$ and $\left(\bar{T}_{y z}\right)_{\zeta= \pm 1}$ are illustrated in Figures 6-9. 


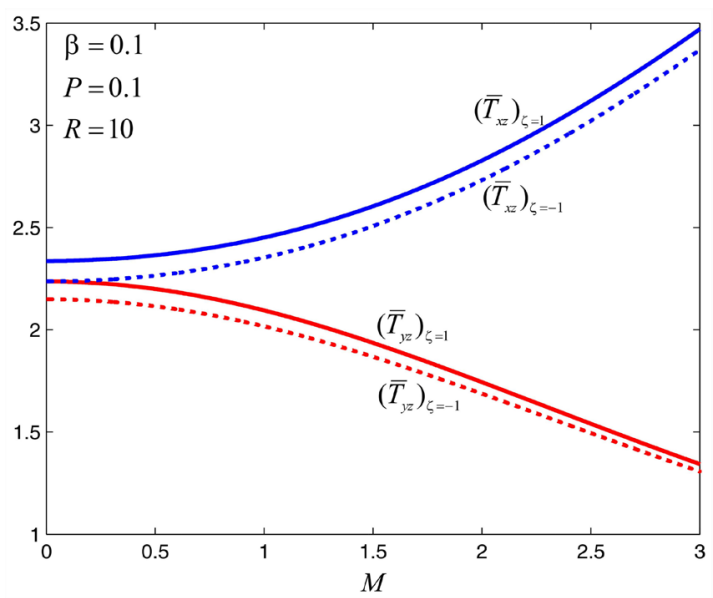

Figure 6. Variations of $\left(\bar{T}_{x z}\right)_{\zeta= \pm 1}$ and $\left(\bar{T}_{y z}\right)_{\zeta= \pm 1}$ with $M(\beta=0.1, P=0.1, R=10)$.

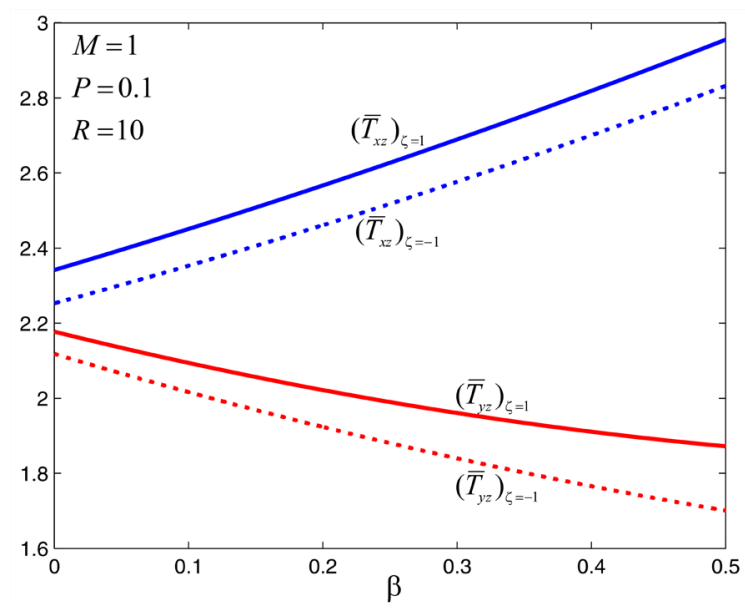

Figure 7. Variations of $\left(\bar{T}_{x z}\right)_{\zeta= \pm 1}$ and $\left(\bar{T}_{y z}\right)_{\zeta= \pm 1}$ with $\beta(M=1, P=0.1, R=10)$.

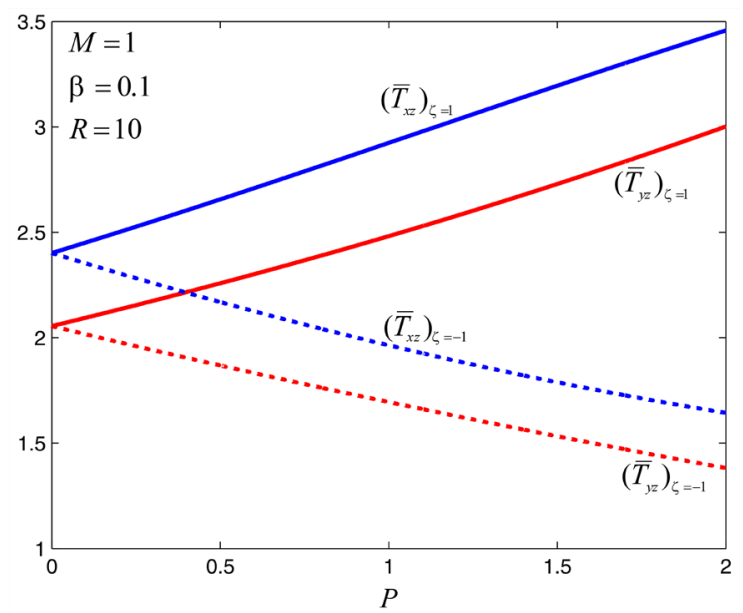

Figure 8. Variations of $\left(\bar{T}_{x z}\right)_{\zeta= \pm 1}$ and $\left(\bar{T}_{y z}\right)_{\zeta= \pm 1}$ with $P(M=1, \beta=0.1, R=10)$. 


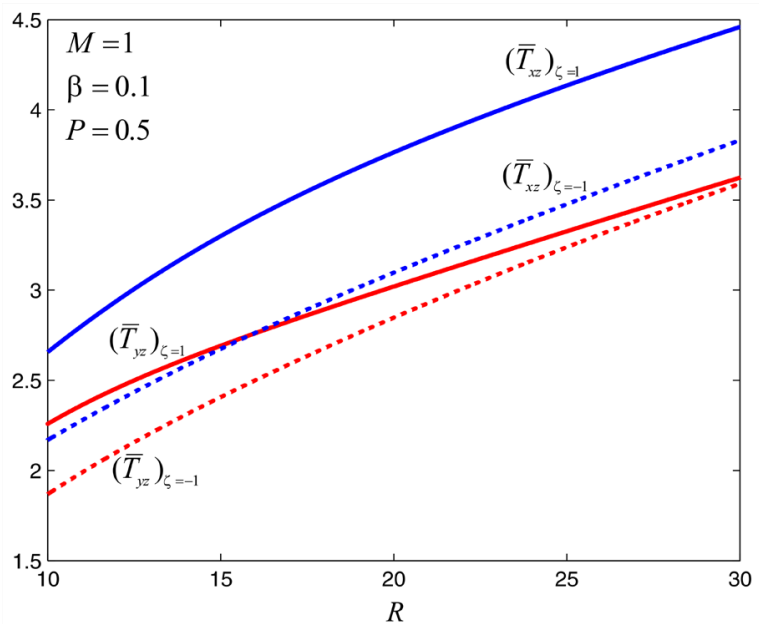

Figure 9. Variations of $\left(\bar{T}_{x z}\right)_{\zeta= \pm 1}$ and $\left(\bar{T}_{y z}\right)_{\zeta= \pm 1}$ with $R$ $(M=1, \beta=0.1, P=0.5)$.

\section{Conclusions}

The conclusions that can be drawn from the performed analysis are pointed out as follows:

- When the Hartmann number $M$ increases, the curves about which the fluid layers rotate start to get closer to the $z$-axis. These curves are closer to the plane $x=0$ than the plane $y=0$. The dimensionless force in the $x$-direction increases but that in the $y$-direction decreases.

- When the second-grade fluid parameter $\beta$ increases, the curves mentioned tend to move away from the $z$-axis. The effect of $\beta$ on the translational velocity in the $y$-direction is greater than that in the $x$-direction. The $x$-component of the dimensionless force increases but the $y$-component decreases. It may be noticed that the change is almost linear.

- The functions $\bar{f}(\zeta)$ and $\bar{g}(\zeta)$ representing the dimensionless $x$ - and $y$-components of the translational velocity vector are anti-symmetric for non-porous disks; however, they are not anti-symmetric for porous disks. When the suction/injection velocity parameter $P$ increases, the space curves get closer to the plane $y=0$ in the region between the top disk and the plane $z=0$, but move away from the plane $x=0$. An adverse effect is observed in the region between the bottom disk and the plane $z=0$. Both the $x$ - and $y$-components of the force on the top disk increase but those on the bottom disk decrease. It is observed that the change is almost linear.

- When the Reynolds number $R$ increases, the space curves in the core region become closer to the $z$-axis, and thus the boundary layers developing on the disks lead to an increase in the horizontal force on the top and bottom disks.

\section{References}

[1] Maxwell, B. and Chartoff, R.P. (1965) Studies of a Polymer Melt in an Orthogonal Rheometer. Transactions of the Society of Rheology, 9, 41-52. 
https://doi.org/10.1122/1.548979

[2] Abbott, T.N.G. and Walters, K. (1970) Rheometrical Flow Systems: Part2. Theory for the Orthogonal Rheometer, Including an Exact Solution of the Navier-Stokes Equations. Journal of Fluid Mechanics, 40, 205-213. https://doi.org/10.1017/S0022112070000125

[3] Rajagopal, K.R. and Gupta, A.S. (1981) Flow and Stability of a Second Grade Fluid between Two Parallel Plates Rotating about Noncoincident Axes. International Journal of Engineering Science, 19, 1401-1409. https://doi.org/10.1016/0020-7225(81)90037-9

[4] Rajagopal, K.R. (1981) The Flow of a Second Order Fluid between Rotating Parallel Plates. Journal of Non-Newtonian Fluid Mechanics, 9, 185-190. https://doi.org/10.1016/0377-0257(87)87015-5

[5] Rajagopal, K.R. (1982) On the Flow of a Simple Fluid in an Orthogonal Rheometer. Archive for Rational Mechanics and Analysis, 79, 39-47. https://link.springer.com/article/10.1007/BF02416565 https://doi.org/10.1007/BF02416565

[6] Mohanty, H.K. (1972) Hydromagnetic Flow between Two Rotating Disks with Noncoincident Parallel Axes of Rotation. Physics of Fluids, 15, 1456-1458. https://doi.org/10.1063/1.1694107

[7] Rao, R. and Rao, P.R. (1985) MHD Flow of a Second Grade Fluid in an Orthogonal Rheometer. International Journal of Engineering Science, 23, 1387-1395. https://doi.org/10.1016/0020-7225(85)90117-X

[8] Ersoy, H.V. (1999) MHD Flow of an Oldroyd-B Fluid between Eccentric Rotating Disks. International Journal of Engineering Science, 37, 1973-1984. https://doi.org/10.1016/S0020-7225(99)00010-5

[9] Guria, M., Das, B.K., Jana, R.N. and Imrak, C.E. (2008) Hydromagnetic Flow between Two Porous Disks Rotating about Non-Coincident Axes. Acta Mechanica Sinica, 24, 489-496. http://link.springer.com/article/10.1007/s10409-008-0158-x

[10] Siddiqui, A.M., Rana, M.A. and Ahmed, N. (2010) Magnetohyrdrodynamics Flow of a Burgers' Fluid in an Orthogonal Rheometer. Applied Mathematical Modelling, 34, 2881-2892. https://doi.org/10.1016/j.apm.2009.12.022

[11] Ersoy, H.V. (2015) Flow of a Maxwell Fluid in a Porous Orthogonal Rheometer under the Effect of a Magnetic Field. American Journal of Mechanical Engineering, 3, 16-20. http://pubs.sciepub.com/ajme/3/1/3/ https://doi.org/10.12691/ajme-3-1-3

[12] Rivlin, R.S. and Ericksen, J.L. (1955) Stress-Deformation Relations for Isotropic Materials. Journal of Rational Mechanics and Analysis, 4, 323-425. https://doi.org/10.1512/iumj.1955.4.54011

[13] Dunn, J.E. and Fosdick, R.L. (1974) Thermodynamics, Stability and Boundedness of Fluids of Complexity 2 and Fluids of Second Grade. Archive for Rational Mechanics and Analysis, 56, 191-252. http://link.springer.com/article/10.1007/BF00280970 https://doi.org/10.1007/BF00280970

[14] Dunn, J.E. and Rajagopal, K.R. (1995) Fluids of Differential Type: Critical Review and Thermodynamic Analysis. International Journal of Engineering Science, 33, 689-729. https://doi.org/10.1016/0020-7225(94)00078-X

[15] Fosdisk, R.L. and Rajagopal, K.R. (1979) Anomalous Features in the Model of Second Order Fluids. Archive for Rational Mechanics and Analysis, 70, 145-152. http://link.springer.com/article/10.1007/BF00250351 https://doi.org/10.1007/BF00250351 